\title{
ALGEBRAIC ORDERS AND CHORDAL LIMIT ALGEBRAS
}

\author{
by ALLAN P. DONSIG*
}

(Received 22nd July 1996)

\begin{abstract}
We develop an isomorphism invariant for limit algebras: an extension of Power's strong algebraic order on the scale of the $K_{0}$-group (Power, $J$. Operator Theory 27 (1992), 87-106). This invariant is complete for a certain family of limit algebras: inductive limits of digraph algebras (a.k.a. finite dimensional CSL algebras) satisfying two conditions: (1) the inclusions of the digraph algebras respect the order-preserving normalisers, and (2) the digraph algebras have chordal digraphs. The first condition is also used to show that the invariant depends only on the limit algebra and not the direct system. We give an intrinsic characterisation of the limit algebra and not the direct system. We give an intrinsic characterisation of the limit algebras satisfying both (1) and (2).
\end{abstract}

1991 Mathematics subject classification: 47D25, 46K50.

A limit algebra is the inductive limit of a direct system

$$
A_{1} \stackrel{x_{1}}{\longrightarrow} A_{2} \stackrel{x_{2}}{\rightarrow} A_{3} \cdots
$$

where the $A_{i}$ are digraph algebras (also called finite dimensional CSL algebras or incidence algebras) and the $\alpha_{i}$ are $*$-extendible embeddings. Different sequences can have the same inductive limits (meaning isometrically isomorphic algebras), so two natural problems arise:

- find isomorphism invariants for a family of limit algebras, and

- find intrinsic properties that characterise a given family.

This pair of problems has motivated much of the work on limit algebras $[5,9,10,13$ 14].

The spectrum, a topological binary relation, is a complete invariant for triangular limit algebras [14]. It is an open question if the spectrum is an invariant for nontriangular limit algebras: at the moment, the spectrum is only known to be an invariant for the direct system $\left(A_{i}, \alpha_{i}\right)$ or equivalently, for the pair $(\mathcal{A}, \mathcal{D})$ of limit algebra and canonical masa. (A canonical masa in $\mathcal{A}$ is, in essence, a limit of diagonal matrices in the $A_{i}$ such that its normalising partial isometries span the algebra.) If the limit algebra

* Partially supported by an NSERC of Canada Postdoctoral Fellowship. 
is selfadjoint, i.e., an $\mathrm{AF} \mathrm{C}^{*}$-algebra, then two canonical masas are conjugate by an approximation inner automorphism (see, for example, [16, Theorem 5.7]). However, there are limit algebras, necessarily non-selfadjoint, containing canonical masas that are not conjugate by an approximately inner automorphism [6]. If, for limit algebras in a particular family, we know that any two such masas in the limit algebra are conjugate by an automorphism, then the spectrum is an invariant; for example, [17, Theorem 4.1] shows such masas are conjugate if the limit algebra is the tensor product of an AF $\mathrm{C}^{*}$-algebra and a digraph algebra.

One can also construct homology groups for the pair $(\mathcal{A}, \mathcal{D})[17]$ and these have been used for classifications of various limit algebras and direct systems [7, 17].

Another invariant for limit algebras, based on the $K_{0}$-group, was introduced by Power in [15]. The $K_{0}$-group and its scale see only the selfadjoint part of the algebra, even when the definition is extended from $\mathrm{C}^{*}$-algebras to limit algebras. However, putting an order on the scale describes the non-selfadjoint part. Following [13], define the diagonal order on projections by saying $p$ is less than $q$ if there is a partial isometry, $w$, that normalises the diagonal of the algebras and so that $w^{*} w=q$ and $w w^{*}=p$. This induces a well-defined ordering, $S(\mathcal{A})$, on the scale of the $K_{0}$-group, called the algebraic order [15]. In [15] Power also considered a second order, the strong algebraic order $S_{1}(\mathcal{A})$, where an additional condition is imposed on the partial isometries $w$ : namely, conjugation by $w$ preserves the diagonal ordering on projections. Such partial isometries are called order-preserving in [5]. In other words, if $\Sigma\left(\mathcal{A} \cap \mathcal{A}^{*}\right)$ is the scale in $K_{0}\left(\mathcal{A} \cap \mathcal{A}^{*}\right)$ and $[p]$ is the Murray-von Neumann equivalence class of the projection $p$, then $S_{1}(\mathcal{A}) \subset \Sigma\left(\mathcal{A} \cap \mathcal{A}^{*}\right) \times \Sigma\left(\mathcal{A} \cap \mathcal{A}^{*}\right)$ denotes the set of elements ([p], [q]) for which there is some $w \in N_{D}^{\text {ord }}(\mathcal{A})$ with $w w^{*}=p$ and $w^{*} w=q$; it is easy to check that this is well-defined.

We add additional information to the strong algebraic order by replacing each pair of equivalent classes of projections, $([p],[q])$, with a triple $([p],[q],[r])$ where $p$ is less than $q$ in the strong algebraic order and $r$ is a common subprojection of $p$ and $q$. Precisely, define

$$
\begin{aligned}
& S_{f}(\mathcal{A})=\left\{([p],[q][r]): \text { there is } w \in N_{D}^{\text {ord }}(\mathcal{A}) \text { with } w^{*} w=q, w w^{*}=p\right. \\
& \text { and } r \text { is the largest subprojection of } p \text { and } q \text { so that } w r=r w=r .\}
\end{aligned}
$$

We call $S_{f}(\mathcal{A})$ the fixed-point algebraic order.

The main result of this paper is that the scaled $K_{0}$-group $K_{0}\left(\mathcal{A} \cap \mathcal{A}^{*}\right)$ together with $S_{f}(\mathcal{A})$ is a complete invariant for limit algebras $\mathcal{A}=\lim \left(A_{i}, \alpha_{i}\right)$ where

(1) the $\alpha_{i}$ send order-preserving elements to order-preserving elements, and

(2) the $A_{i}$ have chordal digraphs.

Direct systems satisfying the second condition have been characterised by Thelwall [18] in terms of the spectrum of the limit algebra. Combining this result with a characterisation of the first condition gives an intrinsic characterisation of this family of limit algebras. 
Condition (1) is natural, since such partial isometries are used in our invariant. If the $A_{i}$ are maximal triangular, i.e., a direct sum of upper-triangle matrix algebras, then the $\alpha_{i}$ satisfying (1) are direct sums of refinement embeddings; such limit algebras have been studied in [5]. Thus, our primary interest here is for algebras $A_{i}$ which are not maximal triangular.

One consequence of condition (1), not previously mentioned, is that the invariants $S_{1}$ and $S_{f}$ do not depend on whether the partial isometries normalise either $\mathcal{A} \cap \mathcal{A}^{*}$ or a canonical masa in $\mathcal{A}$. As a result, we have an invariant that is independent of the choice of $\mathcal{D}$, without losing the convenience of working with partial isometries that normalise the masa $\mathcal{D}$.

Condition (2) is perhaps unexpected. A graph is chordal if every cycle of length more than three has a chord, and we call a digraph chordal if the underlying graph is chordal. (Recall that a cycle of length $n$ is a sequence $\left(v_{1}, e_{1}, v_{2}, e_{2}, \ldots, e_{n}\right)$ where $v_{1}, \ldots, v_{n}$ are $n$ distinct vertices, each $e_{i}$ is a distinct edge with ends $v_{i}$ and $v_{i+1}$, except $e_{n}$, which has ends $v_{n}$ and $v_{1}$. A chord is an edge between some $v_{i}$ and $v_{j}$ not joined by an $e_{k}$.)

In particular, Paulsen, Power and Smith [12] showed that, for digraph algebras with chordal digraphs, all contractive representations are completely contractive, and so admit *-dilations. Muhly and Solel [11] extended this result to 'coordinatised' subalgebras of hyperfinite von Neumann algebras. Thelwall [18] extended Paulsen, Power and Smith's work to limit algebras with chordal spectrum, by showing that such a limit algebra is the limit of a direct system satisfying (2). Applying [12] to each algebra in the system then shows that contractive representations are completely contractive.

Much of this work was done at Lancaster University and I would like to thank the Department of Mathematics \& Statistics for its hospitality; in particular, I would like to thank Steve Power for helpful conversations. I would also like to thank Tim Hudson for his comments on a draft of the paper.

\section{Digraph algebras and normalisers}

To begin, we study normalising partial isometries in digraph algebras. Our focus is on elements that normalise either the algebra intersected with its adjoint or a specified masa in the algebra. The distinction between these choices in limit algebras motivates our concern here.

Definition 1.1. Suppose $A$ is a digraph algebra and $D \subseteq A$ is a selfadjoint subalgebra. Then

$$
N_{D}(A) \stackrel{\text { def }}{=}\left\{x \in A: x \text { is a partial isometry and } x^{*} d x, x d x^{*} \in D \text { for all } d \in D\right\} .
$$

Call $x \in N_{D}(A)$ minimal if $x^{*} x$ and $x x^{*}$ are minimal projections. We abbreviate $N_{\text {AnA. }}(A)$ to $N(A)$. 
The smallest and largest reasonable choices for $D$ are, respectively, $D$ a masa in $A$ and $D$ equal to $A \cap A^{*}$. If $D$ is a masa, then the elements of $N_{D}(A)$ are closely tied to matrix units; precisely, they are sums of unimodular multiples of matrix units, where the matrix units in the sum have orthogonal initial and final projections. However, $N_{D}(A)$ then depends, at least formally, on the choice of the masa $D$. This is not an issue for $A$ a digraph algebra, as all masas in $A$ are unitarily equivalent, but will be important when we turn to limit algebras.

In general $N(A)$ neither contains nor is contained in $N_{D}(A)$ for $D$ a masa in $A$.

\section{Example 1.2. Consider}

$$
A=\left[\begin{array}{cccc}
* & * & * & * \\
* & * & * & * \\
& & * & * \\
& & * & *
\end{array}\right] \text { and } x=\left[\begin{array}{cccc}
0 & 0 & 1 / \sqrt{2} & 1 / \sqrt{2} \\
0 & 0 & -1 / \sqrt{2} & 1 / \sqrt{2} \\
& & 0 & 0 \\
& & 0 & 0
\end{array}\right]
$$

and $D$ is the diagonal matrices. Then $x$ is in $N(A)$ but not in $N_{D}(A)$. Of course, there is a unitary $U \in A \cap A^{*}$ so that $U x U^{*} \in N_{D}(A)$. On the other hand, if

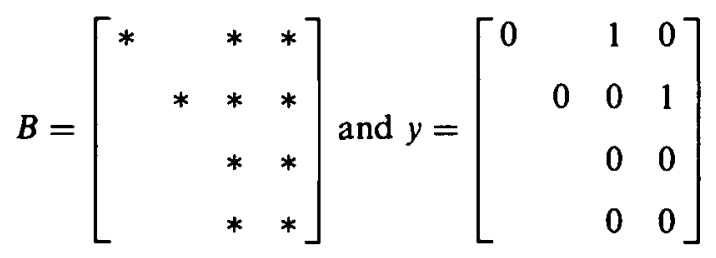

and $D$ is the diagonal matrices, then $y$ is in $N_{D}(B)$ but not in $N(B)$. Since $y$ is a sum of elements in $N(B)$, it may seem that the difficulty above can be overcome by using the span of $N(B)$ but this does not work for limit algebras, as we will show.

Normalising elements with an additional property, order-preservation, are better behaved.

Definition 1.3. Suppose $A$ is a digraph algebra and $D \subseteq A$, a selfadjoint subalgebra. If $p, q \in D$ are projections, then we write $p \prec_{D} q$ if there is some $x \in N_{D}(A)$ so that $x x^{*}=p$ and $x^{*} x=q$. This ordering is reflexive and transitive; it is anti-symmetric if $A \cap A^{*}=D$ and is symmetric if $A=A^{*}$. Note that we use $<$ and $\leq$ for the usual ordering on projections.

We say $x \in N_{D}(A)$ is order-preserving if the map $p \mapsto x p x^{*}$ from $\left\{p \leq x^{*} x\right\}$ to $\left\{p \leq x x^{*}\right\}$ preserves the diagonal order. Then

$$
N_{D}^{\text {ord }}(A) \stackrel{\text { def }}{=}\left\{x \in N_{D}(A): x \text { is order-preserving }\right\}
$$


We use $\prec$ for $\prec_{A \cap A^{*}}$ and $N^{\text {ord }}(A)$ for $N_{A \cap a^{*}}^{\text {ord }}(A)$.

Every minimal element of $N(A)$ is in $N^{\text {ord }}(A)$ so in Example $1.2 x$ is in $N^{\text {ord }}(A)$ while $y$ is not in $N_{D}^{\text {ord }}(B)$. In general, we have the following relation between $N^{\text {ord }}(A)$ and $N_{D}^{\text {ord }}(A)$.

Lemma 1.4. If $A$ is a digraph algebra and $D$ is a masa in $A$, then

$$
\begin{aligned}
N^{\text {ord }}(A) & =\bigcup\left\{N_{E}^{\text {ord }}(A): E \text { is a masa in } A\right\} \\
& =\bigcup\left\{U N_{D}^{\text {ord }}(A) U^{*}: U \text { is a unitary in } A \cap A^{*}\right\} .
\end{aligned}
$$

Proof. Suppose $x \in N_{E}^{o r d}(A)$, for some masa $E$. If $p$ is a projection in $A \cap A^{*}$ with $p \leq x^{*} x$, then $p$ can be written as a linear combination of partial isometries in $N_{E}^{\text {ord }}(A)$. As conjugation by $x$ carries these partial isometries to partial isometries in $A$, it follows that $x p x^{*}$ is also in $A$. Being selfadjoint, $x p x^{*}$ is in $A^{*}$ also and hence $x \in N(A)$. As $x$ is order preserving by hypothesis, we're done.

Conversely, if $x \in N^{\text {ord }}(A)$, then it is straightforward to construct a masa $E$ so that $p \mapsto x p x^{*}$ maps $E$ into itself. Indeed, if $D_{1}$ is a masa in $x^{*} x A x^{*} x$, then conjugation by $x$ carries this to a masa $D_{2}$ in $x x^{*} A x x^{*}$. Now extend the abelian algebra $D_{1} \oplus D_{2}$ to a masa $E$. By construction, $x \in N_{E}^{\text {ord }}(A)$.

The second equality follows easily, as any masa $E$ is unitarily equivalent to $D$ in $A$.

To finish the section, we relate the order-preserving normaliser of a digraph algebra to its reduced digraph, which we now define. Recall that if $A$ is a digraph algebra, then the associated digraph, $G(A)$, has vertices the minimal diagonal projections, $p_{1}, \ldots, p_{n}$, and edges given by

$$
\left(p_{i}, p_{j}\right) \in G(A) \text { if and only if } p_{i} A p_{j} \neq 0 \text {. }
$$

This graph is reflexive and transitive.

Definition 1.5. The reduced digraph associated to a digraph algebra $A$, denoted $G_{r}(A)$, is $G(A) / \approx$ where $p_{i} \approx p_{j}$ if $\left(p_{i}, p_{j}\right)$ and $\left(p_{j}, p_{i}\right)$ are edges of $G(A)$. This digraph, $G_{r}(A)$, is a partial order on $\left\{p_{1}, \ldots, p_{n}\right\} / \approx$.

The advantage of the reduced digraph is that its vertices correspond to the summands of $A \cap A^{*}$. Thus, $G_{r}(A)$ reflects only the non-selfadjoint structure of $A$ whereas $G(A)$ is encumbered with information about $A \cap A^{*}$. Put an equivalence relation on $N^{\text {ord }}(A)$ by setting $x \sim y$ if $x=p y q$ for some partial isometries $p, q \in A \cap A^{*}$ (such $p, q$ are necessarily in $N^{\text {ord }}(A)$ ). It is easy to prove the following fact, which we record for future reference. 
Lemma 1.6. Let $A$ be a digraph algebra. There is a bijection between the edges of $G_{r}(A)$ and the equivalence classes (under $\sim$ ) of minimal elements of $N^{\text {ord }}(A)$.

\section{Order preserving embeddings}

We describe the homomorphisms of digraph algebras that preserve the normalisers of the previous section. It follows that for a limit algebra $\mathcal{A}$, the strong algebraic order $S_{1}(\mathcal{A})$ and the fixed-point strong algebraic order $S_{f}(\mathcal{A})$ are independent of the choice of a canonical masa.

Definition 2.1. Let $A, B$ be digraph algebras with $D \subseteq A$ and $E \subseteq B$ specified selfadjoint subalgebras. Call an algebra homomorphism $\phi: A \rightarrow B$ an embedding if $\phi$ extends to an injective $*$-homomorphism between the generated $C^{*}$-algebras (and so is necessarily isometric) and $\phi\left(N_{D}(A)\right) \subseteq N_{E}(B)$.

Usually $D$ and $E$ are masas. One could also set $D$ to $A \cap A^{*}$ and $E$ to $B \cap B^{*}$. These choices give different families of embeddings. Motivated by Example 1.2, consider

$$
\phi: T_{2} \rightarrow A=\left[\begin{array}{cccc}
* & * & * & * \\
* & * & * & * \\
& * & * \\
& * & *
\end{array}\right]:\left[\begin{array}{ll}
a & b \\
& c
\end{array}\right] \rightarrow\left[\begin{array}{cccc}
a & 0 & b / \sqrt{2} & b / \sqrt{2} \\
0 & a & -b / \sqrt{2} & b / \sqrt{2} \\
& c & 0 \\
& & 0 & c
\end{array}\right]
$$

and

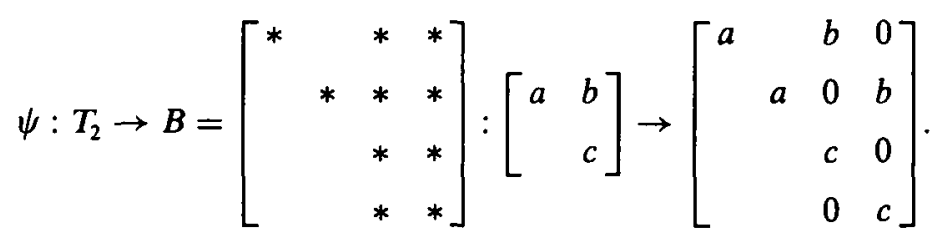

Then $\quad \phi\left(N\left(T_{2}\right)\right) \subset N(A) \quad$ but $\quad \phi\left(N_{D_{2}}\left(T_{2}\right)\right) \not \subset N_{D_{4}}(A) \quad$ while $\quad \psi\left(N_{D_{2}}\left(T_{2}\right)\right) \subset N_{D_{4}}(B)$ but $\psi\left(N\left(T_{2}\right)\right) \not \subset N(B)$. However, if we ask that $N_{D}^{\text {ord }}\left(T_{2}\right)$ be mapped into $N_{E}^{\text {ord }}(A)$, then both choices of $D$ and $E$ give the same family of embeddings and similarly with $B$ instead of $A$.

Lemma 2.2. Let $A, B$ be digraph algebras and $\phi: A \rightarrow B$ a *-extendible algebra homomorphism. Then $\phi\left(N^{\text {ord }}(A)\right) \subseteq N^{\text {ord }}(B)$ if and only if there are masas $D \subseteq A$ and $E \subseteq B$ so that $\phi\left(N_{E}^{\text {ord }}(A)\right) \subseteq N_{E}^{\text {ord }}(B)$.

Proof. $\left(\Longleftrightarrow\right.$ Suppose $x \in N^{\text {ord }}(A)$. By Lemma 1.4 , there is some unitary $U \in A \cap A^{*}$, so that $U x U^{*} \in N_{D}^{\text {ord }}(A)$. By the hypothesis on $\phi$, 


$$
y=\phi(U) \phi(x) \phi(U)^{*}=\phi\left(U x U^{*}\right) \in N_{E}^{\text {ord }}(B) .
$$

If $V=\phi(U)^{*}+1-\phi(1)$, then $\phi(x)=V y V^{*}$ with $V$ unitary and $y \in N_{E}^{\text {ord }}(B)$. Applying Lemma $1.4, \phi(x) \in N^{\text {ord }}(B)$.

$(\Longrightarrow)$ Let $D \subset A$ be a masa. Since $N_{D}^{\text {ord }}(A) \subseteq N^{\text {ord }}(A)$ and $\phi\left(N^{\text {ord }}(A)\right) \subseteq N^{\text {ord }}(B)$, we have $\phi\left(N_{D}^{\text {ord }}(A)\right) \subseteq N^{\text {ord }}(B)$. Hence it suffices to choose $E$ so that $\phi\left(N_{D}^{\text {ord }}(A)\right)$ normalises $E$.

We can choose a system of matrix units $\left\{e_{i j}\right\}$ for $C^{\prime \prime}(A)$ so that $D=\operatorname{span}\left\{e_{i i}\right\}$. It suffices to choose $E$ so that $\phi\left(e_{i j}\right) \in N_{E}(B)$ for all matrix units $e_{i j}$ in $A$. To see this, note that every $x \in N_{D}^{\text {ord }}(A)$ is a sum of unimodular multiples of the $e_{i j}$ 's with orthogonal initial and final projections, say $x=\sum f_{i}$. If $p$ is a projection in $E$ with $p \leq x^{*} x$, then we may write $p=\sum p_{i}$ where $p_{i} \leq f_{i}^{*} f_{i}$. By $\phi$ 's action on matrix units, $\phi\left(f_{i}\right) p_{i} \phi\left(f_{i}\right)^{*} \in E$ for all $i \in I$. However,

$$
\phi(x) p \phi(x)^{*}=\sum \phi\left(f_{i}\right) p_{i} \phi\left(f_{i}\right)^{*}
$$

by orthogonality, so $\phi(x) p \phi(x)^{*} \in E$. Similar arguments work for projections $p \leq x x^{*}$ and conjugation by $\phi(x)^{*}$. Hence $\phi(x) \in N_{E}(B)$.

To construct a masa $E$ in $B$ with $\phi\left(e_{i j}\right) \in N_{E}(B)$ for all matrix units $e_{i j}$ in $A$, first pick one $e_{i i}$ in each summand of $C^{*}(A)$ and write each $\phi\left(e_{i i}\right)$ as a sum of orthogonal minimal projections in $B$. We construct a set of orthogonal minimal projections summing to $\phi(1)$ by looking at images of these minimal projections under conjugation by $\phi(f)$ and $\phi(f)^{*}$ for $f$ a matrix unit with either $f^{*} f=e_{i i}$ in the first case or $f f^{*}=e_{i i}$ in the second. Since $\phi$ is *-extendible, this set is closed under conjugation by $\phi(f)$ for $f$ any matrix unit of $C^{*}(A)$. Now write $1-\phi(1)$ as a sum of orthogonal minimal projections and set $E$ to be the span of all these minimal projections. By construction, $E$ is a masa and $\phi\left(e_{i j}\right) \in N_{E}(B)$ for all matrix units $e_{i j}$.

The same argument also proves the following lemma.

Lemma 2.3. Let $A, B$ be digraph algebras and $\phi: A \rightarrow B$ a *-extendible algebra homomorphism. Then $\phi$ maps the minimal elements of $N^{\text {ord }}(A)$ into $N^{\text {ord }}(B)$ if and only if there are masas $D \subseteq A$ and $E \subseteq B$ so that $\phi$ maps the minimal elements of $N_{D}^{\text {ord }}(A)$ into $N_{E}^{\text {ord }}(B)$.

Definition 2.4. Let $A, B$ be digraph algebras and $D \subseteq A$ and $E \subseteq B$ be selfadjoint subalgebras. Call an algebra homomorphism $\phi: A \rightarrow B$ an order-preserving embedding if it is an embedding and $\phi\left(N_{D}^{\text {ord }}(A)\right) \subseteq N_{E}^{\text {ord }}(B)$. Call $\phi$ a locally order-preserving embedding if it is an embedding and $\phi$ maps minimal elements of $N_{D}^{\text {ord }}(A)$ into $N_{E}^{\text {ord }}(B)$.

These concepts were introduced in [15]. By the previous lemmas, they do not depend on the choice of $D$ and $E$. The following pair of examples shows, first, that a general 
embedding need not be order-preserving and, second, that a locally order-preserving embedding need not be order-preserving;

$$
\psi_{1}: T_{2} \rightarrow T_{4}:\left[\begin{array}{ll}
a & b \\
& c
\end{array}\right] \rightarrow\left[\begin{array}{llll}
a & 0 & 0 & b \\
& a & b & 0 \\
& & c & 0 \\
& & & c
\end{array}\right]
$$

has $e_{12} \in N^{\text {ord }}\left(T_{2}\right)$ but $\psi_{1}\left(e_{12}\right) \notin N^{\text {ord }}\left(T_{4}\right)$, while

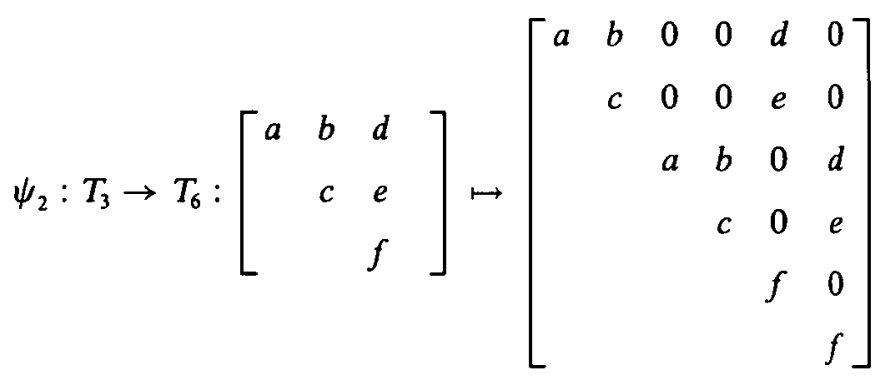

has $e_{11}+e_{23} \in N^{\text {ord }}\left(T_{3}\right)$ and its image is not in $N^{\text {ord }}\left(T_{6}\right)$.

In [5], the order-preserving embeddings between maximal triangular digraph algebras were shown to be direct sums of refinement embeddings. A refinement embedding $\rho_{k}: T_{n} \rightarrow T_{k n}$ is the restriction of the map $M_{n} \rightarrow M_{n k}=M_{n} \otimes M_{k}$ given by $a \mapsto a \otimes 1_{k}$. This was used in [5] to obtain a classification of limit algebras $\underset{\lim }{\rightarrow}\left(T_{n_{i}} \alpha_{i}\right)$ with each $\alpha_{i}$ an order-preserving embedding and to construct an invariant $\overrightarrow{\text { for }}$ the analogous limit algebras where the $T_{n_{i}}$ are replaced with direct sums of upper-triangular matrix algebras.

A masa $\mathcal{D}$ in an AF $C^{*}$-algebra $\mathcal{C}$ is a canonical masa if there is a nested sequence of finite dimensional subalgebras of $\mathcal{C}$, say $\left(C_{i}\right)$, so that

(1) $\mathcal{C}=\overline{\cup_{i} C_{i}}$,

(2) $D_{i}=\mathcal{D} \cap C_{i}$ is a masa in $C_{i}$ for all $i$, and

(3) $N_{D_{i}}\left(C_{i}\right)$ is contained in $N_{D_{i+1}}\left(C_{i+1}\right)$ for all $i$.

In other words, $\mathcal{C}$ is the inductive limit of finite dimensional $\mathrm{C}^{*}$-algebras with respect to embeddings and $\mathcal{C}$ is spanned by $N_{\mathcal{D}}(\mathcal{C})$, where $\mathcal{D}$ is the limit of masas in the finite dimensional $C^{*}$-algebras.

Suppose $\mathcal{A}$ is a subalgebra of an $\mathrm{AF} \mathrm{C}^{*}$-algebra containing a canonical masa D. Equivalently, consider $\mathcal{A}=\lim \left(A_{i}, \alpha_{i}\right)$ with $\alpha_{i}\left(N_{D_{i}}\left(A_{i}\right)\right) \subseteq N_{D_{i+1}}\left(A_{i+1}\right)$. Although span $N_{D_{i}}\left(A_{i}\right)=\operatorname{span} N\left(A_{i}\right)$ for each $\vec{i}$, it does not follow that span $N_{\mathcal{D}}(\mathcal{A})=\operatorname{span} N(\mathcal{A})$. The following example is from [17] where this issue and its implications for defining homology groups are discussed. Let $\mathcal{C}$ be the $U H F\left(2^{\infty}\right) C^{*}$-algebra and $\mathcal{B}$ a canonical masa in $\mathcal{C}$. Then the algebra 


$$
A=\left[\begin{array}{ll}
\mathcal{B} & \mathcal{C} \\
0 & \mathcal{C}
\end{array}\right]
$$

will have span $N(\mathcal{A})=\mathcal{A} \cap \mathcal{A}^{*}$, although $\operatorname{span} N_{\mathcal{D}}(\mathcal{A})=\mathcal{A}$ if $\mathcal{D}$ is, say $\mathcal{B} \oplus \mathcal{B}$. However, $N^{\text {ord }}(\mathcal{A})$ and $N_{\mathcal{D}}^{\text {ord }}(\mathcal{A})$ have the same span, albeit $\mathcal{A} \cap \mathcal{A}^{*}$.

We finish the section by showing a close connection between $N^{\text {ord }}(\mathcal{A})$ and $N_{\mathcal{D}}^{\text {ord }}(\mathcal{A})$.

Proposition 2.5. Suppose $\mathcal{A}$ is a subalgebra of an $A F C^{*}$-algebra that contains a canonical masa. Then

$$
N^{\text {ord }}(\mathcal{A})=\bigcup\left\{N_{\mathcal{D}}^{\text {ord }}(\mathcal{A}): \mathcal{D} \subseteq \mathcal{A}, \mathcal{D} \text { is a canonical masa }\right\}
$$

Proof. ( $\supseteq$ ) Let $\mathcal{D} \subseteq \mathcal{A}$ be a canonical masa and $v \in N_{\mathcal{D}}^{\text {ord }}(\mathcal{A})$. Then there is a sequence of subalgebras $\left(A_{i}\right)$ so that $\mathcal{A}=\overline{\cup_{i} A_{i}}$ and $N_{D_{i}}\left(A_{i}\right) \subseteq N_{D_{i+1}}\left(A_{i+1}\right)$ for all $i$, where $D_{i}=\mathcal{D} \cap A_{i}$ is a masa in $A_{i}$.

By [16, Lemma 5.5], $v=d w$ where $d$ is a partial isometry in $\mathcal{D}$ and $w \in N_{D_{k}}\left(A_{k}\right)$ for some $k$. As $d, v \in N_{D}^{\text {ord }}(\mathcal{A})$, it follows that $w \in N_{D_{i}}^{\text {ord }}\left(A_{i}\right)$ for all $i \geq k$. Hence by Lemma $1.4, w$ is in $N^{\text {ord }}\left(A_{i}\right)$ for $i \geq k$ and so is in $N^{\text {ord }}(\mathcal{A})$.

() Suppose that $v \in N^{\text {ord }}(\mathcal{A})$; then $p=v v^{*} \prec q=v^{*} v$. By writing $v$ as a sum of elements of $N^{\text {ord }}(A)$, we may assume that $p q=0$. Also, we may suppose $p, q \in \mathcal{E}$, for some canonical masa $\mathcal{E} \subset \mathcal{A}$. While the subalgebra $p \mathcal{E} p$ need not equal $v q \mathcal{E} q v^{*}$, by $[15$, Lemma 2.2] there is $w \in N_{\varepsilon}(\mathcal{A})$ with $w v q \mathcal{E} q v^{*} w^{*}=p \mathcal{E} p$. Let $U=1-p+w^{*}$ and $\mathcal{D}=U \mathcal{E} U^{*}$, a canonical masa in $\mathcal{A}$. Then $U$ is a unitary with $U p \mathcal{E} p U^{*}=v q \mathcal{E} q v^{*}$ and $U q \mathcal{E} q U^{*}=q \mathcal{E} q$, so $v \in N_{D}(\mathcal{A})$. Since conjugation by $v$ preserves the ordering on $\mathcal{A} \cap \mathcal{A}^{*}$, it also preserves the ordering on $\mathcal{D}$, hence $v \in N_{\mathcal{D}}^{\text {ord }}(\mathcal{A})$.

It follows immediately from the next theorem that the invariants $S_{1}$ and $S_{f}$ do not depend on the choice of a canonical masa in the limit algebra. Recall from the introduction that

$$
\begin{aligned}
& S_{f}(\mathcal{A})=\left\{([p],[q],[r]): \text { there is } w \in N_{D}^{\text {ord }}(\mathcal{A}) \text { with } w^{*} w=q, w w^{*}=p\right. \\
& \text { and } r \text { is the largest subprojection of } p \text { and } q \text { so that } w r=r w=r .\} .
\end{aligned}
$$

Theorem 2.6. Let $\mathcal{A}$ be a subalgebra of an $A F C^{*}$-algebra that contains a canonical masa $\mathcal{D}$. If $v \in N^{\text {ord }}(\mathcal{A})$ then there is $u \in N_{\mathcal{D}}^{\text {ord }}(\mathcal{A})$ with $u u^{*}=v v^{*}$ and $u^{*} u=v^{*} v$.

Proof. We can choose a direct system for the pair $(\mathcal{A}, \mathcal{D})$, i.e., finite dimensional algebras $A_{i}$ and embeddings from $A_{i}$ to $A_{i+1}$. Let $p=v v^{*}, q=v^{*} v$. For some sufficiently large $k, p$ and $q$ are Murray-von Neumann equivalent in $A_{k} \cap A_{k}^{*}$ to projections in $\mathcal{D} \cap A_{k}$. As partial isometries in $\mathcal{A} \cap \mathcal{A}^{*}$ are order-preserving, it follows that we can multiply $v$ on either side by these partial isometries and so assume that $p$ and $q$ are in $\mathcal{D}$. 
As $v$ is order-preserving, conjugation by $v$ is an isomorphism from $q \mathcal{A} q$ to $p \mathcal{A} p$. Observing that $p \mathcal{A} q$ is a $p \mathcal{A} p-q \mathcal{A} q$ bimodule, it follows that the compression of $\mathcal{A}$ by $p+q$ contains a subalgebra isomorphic to $p \mathcal{A} p \otimes T_{2}$. This contains the further subalgebra

$$
\mathcal{S}=p\left(\mathcal{A} \cap \mathcal{A}^{*}\right) p \otimes T_{2} \subseteq \mathcal{A}
$$

As $v$ is order-preserving, we may choose the inclusion of $\mathcal{S}$ in $\mathcal{A}$ so that $v$ is in $\mathcal{S}$.

Since $\mathcal{S}$ is the tensor product of an AF $C^{*}$-algebra and a digraph algebra, by [17, Theorem 4.1] any two canonical masas in $\mathcal{S}$ are approximately inner conjugate. In particular, there is some sequence of unitaries in $S,\left(w_{i}\right)$, so that the automorphism

$$
\rho(s)=\lim _{i \rightarrow \infty} w_{i} s w_{i}^{*},
$$

exists for every $s \in \mathcal{S}$ and $\rho$ carries $(p+q) \mathcal{D}(p+q)=p \mathcal{D} p+q \mathcal{D} q$ to $v q \mathcal{D} q v^{*}+q \mathcal{D} q$.

As $p$ and $q$ can be identified with $1 \otimes e_{1,1}$ and $1 \otimes e_{2,2}$ in $\mathcal{S}$, it follows that each $w_{i}$ fixes $p$ and $q$. Hence $\rho(q \mathcal{D} q)=q \mathcal{D} q$ and $\rho\left(v q \mathcal{D} q v^{*}\right)=p \mathcal{D} p$. Thus $u=\rho(v)$ conjugates $q \mathcal{D} q$ to $p \mathcal{D} p$ and so $u$ is in $N_{\mathcal{D}}^{\text {ord }}(\mathcal{A})$ and $u u^{*}=p, u^{*} u=q$, as required.

\section{Completeness of the invariant $S_{f}$}

In this section we prove that the fixed-point algebraic order is a complete isomorphism invariant for limits of digraph algebras with chordal digraphs and order-preserving embeddings. The proof is based on a finite dimensional lifting result, which in turn needs a number of technical lemmas. The first of these lemmas is a generalisation of Lemma 3 in [5].

Lemma 3.1. Let $\phi: A \rightarrow B$ be an embedding between digraph algebras. Then $\phi$ is order-preserving if and only if $\phi(x) \in N^{\text {ord }}(B)$ for all elements $x \in N^{\text {ord }}(A)$ of the form $x=f_{1}$ or $x=f_{1}+f_{2}$ where $f_{i}$ is a minimal element of $N^{\text {ord }}(A)$.

If $A$ is chordal, then we need only consider elements $x$ of the form $x=f$ or $x=p+f$, where $f$ is a minimal element of $N^{\text {ord }}(A)$ and $p$ is a minimal projection in $A \cap A^{*}$.

Proof. One direction of the first statement is trivial. For the other direction, if $\phi$ is not order-preserving, then there is some $y \in N^{\text {ord }}(A)$ so that $z=\phi(y) \notin N^{\text {ord }}(B)$. In particular, there are minimal projections $a, b$ in $B$ so that the diagonal order on $z a z^{*}$ and $z b z^{*}$ is not the same as the diagonal order on $a$ and $b$.

As $z a$ is a summand of $\phi(y)$ and is a minimal element of $N^{\text {ord }}(B)$, there is a minimal element of $f_{1} \in N^{\text {ord }}(A)$ so that $z a$ is a summand of $\phi\left(f_{1}\right)$. If $z b$ is also a summand of $\phi\left(f_{1}\right)$, then we let $x=f_{1}$ and are done. Otherwise, there is a minimal element $f_{2} \in N^{\text {ord }}(A)$ so that $z b$ is a summand of $\phi\left(f_{2}\right)$. Moreover, as $z a$ and $z b$ are both summands of $\phi(y)$, then $f_{1}$ and $f_{2}$ are both summands of $y$ so $x=f_{1}+f_{2} \in N^{\text {ord }}(A)$, yet $\phi(x) \notin N^{\text {ord }}(B)$. 
To prove the final statement, we suppose $A$ is chordal and show that if there is $f_{1}+f_{2} \in N^{\text {ord }}(A)$ with $\phi\left(f_{1}+f_{2}\right) \notin N^{\text {ord }}(B)$, then there is some element $p+f \in N^{\text {ord }}(A)$ with $\phi(p+f) \notin N^{\text {ord }}(B)$, where $p$ is a minimal projection and $f, f_{1}, f_{2}$ are minimal elements of $N^{\text {ord }}(A)$.

If we compress $A$ by the sum of the initial and final projections of $f_{1}$ and $f_{2}$, we obtain a subalgebra of $M_{4}$, call it $C$. Identify $f_{1}+f_{2}$ with $e_{1,3}+e_{2,4}$ in $M_{4}$. This implies, as $f_{1}+f_{2}$ is order-preserving, that the two orthogonal $2 \times 2$ diagonal blocks in $C$ are the same. Note that compressing $B$ by $a+b+z a z^{*}+z b z^{*}$ gives a subalgebra of $M_{4}, D$, with

$$
C \subset D \subset M_{4}
$$

and, with our identification, $e_{1,3}+e_{2,4}$ is not order-preserving in $D$. We consider three cases, as the two isomorphic diagonal blocks of $C$ are either $M_{2}, T_{2}$, or $D_{2}$, the diagonal $2 \times 2$ matrices. In fact, the case $M_{2}$ cannot occur, as there is then no subalgebra $D$ with $C \subset D \subset M_{4}$ and $e_{1,3}+e_{2,4} \notin N^{\text {ord }}(D)$.

For the case $T_{2}, C$ is either $T_{4}$ or $T_{2} \otimes T_{2}$ and $D$ is the span of $C$ and either $e_{2,1}$ or $e_{4,3}$. For example, if we have

$$
C=\left[\begin{array}{rrrr}
* & * & * & * \\
& * & 0 & * \\
& * & * \\
& & & *
\end{array}\right] \subset D=\left[\begin{array}{llll}
* & * & * & * \\
* & * & 0 & * \\
& & * & * \\
& & & *
\end{array}\right]
$$

then $e_{1,1}+e_{2,4}$ is in $N^{\text {ord }}(C)$ but not in $N^{\text {ord }}(D)$, giving the required element. The other possibilities are similar.

For the case $D_{2},\left(e_{1,1}+e_{2,2}\right) C\left(e_{3,3}+e_{4,4}\right)$ can be any subalgebra of $M_{2}$ containing $D_{2}$ except, as $C$ is chordal, $M_{2}$ itself. For example, if $e_{2,3} \notin C$, then $e_{1,3}+e_{2,2}$ and $e_{2,4}+e_{3,3}$ are in $N^{\text {ord }}(C)$. However, if both these elements are also in $N^{\text {ord }}(D)$, then the two orthogonal diagonal blocks in $D$ are the same, contradicting $f_{1}+f_{2} \notin N^{\text {ord }}(D)$. If $e_{1,4} \notin C$, then the argument is similar.

The hypothesis of chordality is essential; the natural inclusion from the digraph algebra

$$
A\left(D_{4}\right)=\left[\begin{array}{llll}
* & 0 & * & * \\
& * & * & * \\
& & * & 0 \\
& & & *
\end{array}\right]
$$

into $T_{4}$ is not order-preserving (consider $e_{1,4}+e_{2,3}$ ) but the order-preserving elements 
of $A\left(D_{4}\right)$ of the form $p+f$, namely sums of two diagonal matrix units, are all orderpreserving elements of $T_{4}$.

Chordal digraphs are contained in the set of interpolating digraphs, introduced in [2]. A digraph is interpolating if every $2 k$-cycle, $k \geq 3$ has a chord and every 4 -cycle has an interpolating vertex, that is, if $a, b, c, d$ are vertices with edges from $a$ and $b$ to $c$ and $d$, then there is another vertex $v$ with edges from $a$ and $b$ to $v$ and from $v$ to $c$ and $d$. The arguments of the previous paragraph apply to the inclusion of

$$
\left[\begin{array}{ccccc}
* & 0 & * & * & * \\
& * & * & * & * \\
& & * & * & * \\
& & & * & 0 \\
& & & & *
\end{array}\right]
$$

in $T_{5}$, so we cannot extend Lemma 3.1 to algebras with interpolating digraphs.

Lemma 3.2. Let $B$ be a digraph algebra, $P$ a projection in $B$ and $X$ an element of $N^{\text {ord }}(B)$ so that $P+X$ is a partial isometry. If there is some $Y \in N^{\text {ord }}(B)$ with the same initial and final projections as $X$ so that $P+Y \in N^{\text {ord }}(B)$, then $P+X \in N^{\text {ord }}(B)$.

Proof. Let $Q=X^{*} X$ and $R=X X^{*}$, both projections in $B$. The initial projection of $P+X$ is $P+Q$ and the final projection is $P+R$.

Suppose $a, b \leq P+Q$ are minimal projections and $a^{\prime}$ is the conjugate of $a$ under $P+X$ and $b^{\prime}$ is the conjugate of $b$. To show $P+X \in N^{\text {ord }}(B)$, it suffices to show that $a<b$ if and only if $a^{\prime}<b^{\prime}$. If $a, b \leq P$, then $a^{\prime}=a$ and $b^{\prime}=b$ so there is nothing to do. If $a, b \leq Q$, then $X \in N^{\text {ord }}(B)$ implies that $a<b$ if and only if $a^{\prime}<b^{\prime}$.

Next we suppose that $a \leq Q, b \leq P$. Then $a^{\prime} \leq R$ and $b^{\prime}=b$. If $a<b$, then since $a^{\prime}<a$ we have $a^{\prime}<b^{\prime}$. It remains only to show that $a^{\prime}<b^{\prime}$ implies $a<b$. Let $S=\vee\{c \leq Q: c<b\}$ and $S^{\prime}=\vee\left\{d \leq R: d \prec b^{\prime}\right\}$. We have just shown that $X S X^{*} \leq S^{\prime}$. If the projections $S$ and $S^{\prime}$ have the same rank, then it follows that $X S X^{*}=S^{\prime}$ which implies $a<b$. However, since $P+Y \in N^{\text {ord }}(B)$ and $b^{\prime}=b$ is fixed under conjugation by $P+Y$, we have $Y S Y^{*}=S^{\prime}$. Hence $S$ and $S^{\prime}$ do have the same rank.

If $a \leq P$ and $b \leq Q$, then we have $b^{\prime}<b$ so $a^{\prime}<b^{\prime}$ implies $a<b$. If $a^{\prime}<b^{\prime}$ then we let $S=\vee\{c \leq Q: a<c\}$ and $S^{\prime}=V\left\{d \leq R: a^{\prime}<d\right\}$ and repeat the argument of the previous paragraph.

Lemma 3.3. Let $B$ be a chordal digraph algebra and let $X, Y \in N^{\text {ord }}(B)$ have the same initial and final projections. Then $X Y^{*} \in B \cap B^{*}$.

Proof. If $B^{\prime}$ is the compression of $B$ by $X X^{*}$, then conjugation by $X Y^{*}$ gives an automorphism of $B^{\prime}$, say $\rho$. Further, if $a, b$ are projections in $B^{\prime}$, then $X, Y \in N^{\text {ord }}(B)$ implies that $a<b$ if and only if $\rho(a) \prec \rho(b)$. As $B^{\prime} \cap\left(B^{\prime}\right)^{*}$ is a direct sum of matrix 
algebras, the factors of $B^{\prime} \cap\left(B^{\prime}\right)^{*}$, this follows $\rho$ induces a permutation of these factors.

Claim: this permutation is the identity.

Observe that the claim implies that $X Y^{*}$ is a sum of elements in the factors of $B^{\prime} \cap\left(B^{\prime}\right)^{*}$ and hence $X Y^{*} \in B \cap B^{*}$. Thus, it suffices to prove the claim.

If the permutation is not the identity, then we can decompose it into cycles. Let $B_{1}, \ldots, B_{k}, k \geq 2$, be distinct factors in $B^{\prime} \cap\left(B^{\prime}\right)^{*}$ so that $\rho\left(B_{i}\right)=B_{i+1 \bmod k}$.

If there were projections $a \in B_{i}, b \in B_{j}$ with $i \neq j$ so that $a \prec b$, then it would follow that $b<a$ and so $B_{i}$ and $B_{j}$ are contained in the same factor of $B^{\prime}$, contradicting our choice of $a$ and $b$. Thus there are no edges in $G_{r}(B)$ between any $B_{i}$ and $B_{j}, i \neq j$. Both $X^{*}$ and $Y^{*}$ carry these factors of $B^{\prime} \cap\left(B^{\prime}\right)$ to the same set of factors of $B \cap B^{*}$, say $C_{1}, \ldots, C_{k}$. And as $X, Y \in N^{\text {ord }}(B)$, there are also no edges in $G_{r}(B)$ between any $C_{i}$ and $C_{j}, i \neq j$.

The subgraph of $G_{r}(B)$ with vertices corresponding to $B_{1}, \ldots, B_{k}, C_{1}, \ldots, C_{k}$ contains the $2 k$-cycle digraph $D_{2 k}$. As $G_{r}(B)$ is chordal, there must be an additional edge in the subgraph, and by the previous paragraph, it must join some $B_{i}$ and $C_{j}$. If $k>2$, then we obtain a smaller cycle digraph and repeating this argument, we eventually obtain factors $B_{i}, B_{j}$ and $C_{m}, C_{n}$ so that the corresponding subgraph of $G_{r}(B)$ is $D_{4}$. But for the 4-cycle digraph, any additional edge must join $B_{i}$ to $B_{j}$ or $C_{m}$ to $C_{n}$, a contradiction proving the claim.

Chordal digraphs have long been known to possess one vertex elimination scheme, the perfect vertex elimination scheme [8]. As chordal digraphs are interpolating, they also possess a vertex elimination scheme established for interpolating digraphs in [2]. That is, in any interpolating digraph, there is a vertex with at most one immediate successor and one immediate predecessor, so that deleting this vertex and all of its associated edges gives a subgraph which is itself interpolating. This provides an effective scheme for proving theorems by induction on the number of vertices of an interpolating digraph.

We use a slight modification of the vertex elimination scheme. Instead of deleting a vertex and its associated edges, we delete only the edges involving the vertex, leaving the vertex isolated. As the vertices correspond to the summands of $A \cap A^{*}$, the inductive step is now between two algebras with the same selfadjoint subalgebra and the base case is now a finite dimensional $\mathrm{C}^{*}$-algebra. To distinguish this construction, we call it an edge elimination scheme.

If $\phi: A \rightarrow B$ is an embedding, we use $\phi_{*}$ to denote the induced map from $K_{0}\left(A \cap A^{*}\right)$ to $K_{0}\left(B \cap B^{*}\right)$.

Theorem 3.4. Suppose that $A$ and $B$ are digraph algebras with chordal digraphs and that $\theta: K_{0}\left(A \cap A^{*}\right) \rightarrow K_{0}\left(B \cap B^{*}\right)$ is a scale-preserving ordered-group morphism. If $\theta$ satisfies $\theta^{(3)}\left(S_{f}(A)\right) \subseteq S_{f}(B)$ and $\theta$ induces an ordered-group morphism from $K_{0} C^{*}(A)$ to $K_{0} C^{*}(B)$, then there is an order-preserving embedding $\phi: A \rightarrow B$ with $\phi_{*}=\theta$.

If $\tau: A \rightarrow B$ is another order-preserving embedding with $\tau_{*}=\theta$, then there is a unitary in $B \cap B^{*}, U$, so that $\tau=\operatorname{Ad} U \circ \phi$. 
Proof. We proceed by induction on the number of proper edges of $G_{r}(A)$, using the edge elimination scheme. If $G_{r}(A)$ contains no proper edges, then $A=A \cap A^{*}=C^{*}(A)$ and the result, including inner conjugacy, then follows from the lifting result for finite dimensional $\mathrm{C}^{*}$-algebras (for example, see [20, Lemma 12.1.2]).

For the inductive step, fix a digraph algebra $A$. By the edge elimination scheme, there is a vertex of $G_{r}(A), v$, that has at most one immediate successor and at most one immediate predecessor. Let $A^{\prime}$ be the subalgebra of $A$ so that $G_{r}\left(A^{\prime}\right)$ is $G_{r}(A)$ with all edges involving $v$ deleted. Then $G_{r}\left(A^{\prime}\right)$ has at least one less proper edge and so, by the inductive hypothesis, the theorem holds for $A^{\prime}$ and we have an order-preserving embedding $\psi: A^{\prime} \rightarrow B$.

To prove the theorem for $A$, it suffices to extend $\psi$ to an order-preserving embedding $\phi$ on $A$ and to show that any other extension is of the form $\operatorname{Ad} U \circ \phi$ for some unitary $U \in B \cap B^{*}$. We prove the existence and uniqueness parts separately.

Existence. Case 1: $v$ has one immediate predecessor and no immediate successor.

There is an additional proper edge in $G_{r}(A)$ from the predecessor to $v$ and by Lemma 1.6, there is an equivalence class of elements of $N^{\text {ord }}(A)$ that corresponds to this additional proper edge. Let $x$ be one element of this class. Once $\phi(x)$ is specified, all other elements of the class are determined, as they are products of $x$ and elements of $A \cap A^{*}$. As $\phi$ agrees with $\psi$ on $A^{\prime}, \phi$ is also determined on $A$.

Since $x x^{*}$ and $x^{*} x$ are in $A \cap A^{*} \subset A^{\prime}$, we need only specify a partial isometry from $\psi\left(x^{*} x\right)$ to $\psi\left(x x^{*}\right)$. As $x \in N^{\text {ord }}(A)$, we have $\left(\left[x x^{*}\right],\left[x^{*} x\right]\right) \in S_{1}(A)$. The restriction of $S_{f}(A)$ equals $S_{1}(A)$ so we have $\theta^{(2)}\left(S_{1}(A)\right) \subseteq S_{1}(B)$. As $\left.\psi\right|_{A \cap A^{*}}=\left.\phi\right|_{A \cap A^{*}}$ induces $\theta$, this fact implies $\left(\left[\psi\left(x x^{*}\right)\right],\left[\psi\left(x^{*} x\right)\right]\right) \in S_{1}(B)$. So there is some $X \in N^{\text {ord }}(B)$ with

$$
X X^{*}=\psi\left(x x^{*}\right) \text { and } X^{*} X=\psi\left(x^{*} x\right)
$$

Let $\phi(x)=X$. As $\phi(x) \in N^{\text {ord }}(B)$ and $x \sim y$ imply $\phi(y) \in N^{\text {ord }}(B)$, it follows that $\phi$ is locally order-preserving.

To show $\phi$ is order-preserving, it suffices, by Lemma 3.1 , to show that $\phi(p+e) \in N^{\text {ord }}(B)$ for all minimal projections $p$ and minimal elements $e \in N^{\text {ord }}(A)$ with $p+e \in N^{\text {ord }}(A)$. Fix such an element $e+p$. Since $e+p \in N^{\text {ord }}(A)$, we have

$$
\left(\left[p+e e^{*}\right],\left[p+e^{*} e\right],[p]\right) \in S_{f}(A) .
$$

Then $\left(\left[\phi\left(p+e e^{*}\right)\right],\left[\phi\left(p+e^{*} e\right)\right],[\phi(p)]\right) \in S_{f}(B)$, so there is some $Y \in N^{\text {ord }}(B)$ so that

$$
P+Y \in N^{\text {ord }}(B), \quad Y Y^{*}=\phi\left(e e^{*}\right) \quad \text { and } \quad Y^{*} Y=\phi\left(e^{*} e\right)
$$

Applying Lemma 3.2 with $P=\phi(p), X=\phi(e)$ and $Y$, it follows that $\phi(p+e)=$ $P+X \in N^{\text {ord }}(B)$, as required. Thus $\phi$ is order-preserving.

Case 2: $v$ has no immediate predecessor and one immediate successor.

This case is dual to Case 1 and the same argument, with only trivial modifications, applies. 
Case 3: $v$ has one immediate predecessor and one immediate successor.

Let $u$ be the predecessor and $w$ the successor. We have two additional proper edges, one from $u$ to $v$ and one from $v$ to $w$. By Lemma 1.6, there are two equivalence classes of minimal elements of $N^{\text {ord }}(A)$ that correspond to these edges. Let $x$ be an element of the first class and $y$ an element of the second, chosen so that $x x^{*}=y^{*} y$. Then $y x \neq 0$ corresponds to the edge from $u$ to $w$ and so is in $A^{\prime}$.

We will specify $\phi(y)$ and $\phi(x)$ in $N^{\text {ord }}(B)$ so that $\phi(y) \phi(x)=\psi(y x)$. As before, this will determine $\phi$ and will imply that $\phi$ is locally order-preserving.

Let $p=y y^{*}$. Note that $p \prec y^{*} y=x x^{*}$ and $x x^{*} \prec x^{*} x$ implies $p \prec x^{*} x$. If $p+x \notin N^{\text {ord }}(A)$, then either $x x^{*} \prec p$ or $x^{*} x \prec p$, which, together with the last sentence, implies $y \in A \cap A^{*}$, contrary to our choice of $y$. Thus $p+x \in N^{\text {ord }}(A)$ and so we have

$$
\left(\left[p+x x^{*}\right],\left[p+x^{*} x\right],[p]\right) \in S_{f}(A) .
$$

Since $\theta^{(3)}\left(S_{f}(A)\right) \subseteq S_{f}(B)$, there is some $X \in N^{\text {ord }}(B)$ so that

$$
\phi(p)+X \in N^{\text {ord }}(B), \quad X X^{*}=\phi\left(x^{*} x\right) \quad \text { and } \quad X^{*} X=\phi\left(x x^{*}\right) .
$$

Let $Y=\psi(y x) X^{*}$, the conjugate of $\psi(y x)$ under $\phi(p)+X$. As $\phi(p)+X \in N^{\text {ord }}(B), Y$ is in $B$. Also, $Y$ is in $N^{\text {ord }}(B)$ since both $X$ and $\psi(x y)$ are in $N^{\text {ord }}(B)$.

Let $\phi(x)=X$ and $\phi(y)=Y$. Clearly, $\phi(y) \phi(x)=\psi(y x)$. To show $\phi$ is orderpreserving, we repeat the corresponding argument of Case 1.

Uniqueness. Suppose now that $\tau: A \rightarrow B$ is another order-preserving map with $\tau_{*}=\theta$. By the inductive hypothesis, there is a unitary $U \in B^{*} \cap B$ so that $\left.\operatorname{Ad} U \circ \tau\right|_{A^{\prime}}$ equals $\psi$. Thus, we may suppose that $\tau=\phi$ on $A^{\prime}$. Let $A_{v}$ be the factor, i.e., matrix summand, of $A \cap A^{*}$ corresponding to $v \in G_{r}(A)$.

Case 1: $v$ has one immediate predecessor and no immediate successor.

It suffices to find a partial isometry $V \in B \cap B^{*}$ with $V^{*} V=V V^{*}=\phi\left(1_{A_{v}}\right)$ so that

$$
V \tau(x)=\phi(x)
$$

for all $x$ minimal elements of $N^{\text {ord }}(A)$ with initial projections in $A_{v}$. Indeed, since each minimal element of $N^{\text {ord }}(A)$ not in $A^{\prime}$, say $z$, is a product $x y$ where $y \in N^{\text {ord }}\left(A^{\prime}\right)$ and $x$ is a minimal element of $N^{\text {ord }}(A)$ with initial projection in $A_{v}$, it follows that $V \tau(z)=\phi(z)$. Hence $U=1_{B}-\phi\left(1_{A_{0}}\right)+V$ will satisfy $\operatorname{Ad} U \circ \tau=\phi$, since $N^{\text {ord }}(A)$ spans $A$ and every element of $N^{\text {ord }}(A)$ is a sum of minimal elements. As $U \in B \cap B^{*}$, Ad $U$ maps $B$ to $B$ and is order-preserving.

Let $x$ be a minimal element of $N^{\text {ord }}(A)$ in the equivalence class corresponding to the additional edge of $G_{r}(A)$. Then $\tau(x)$ and $\phi(x)$ are both in $N^{\text {ord }}(B)$ and have the same initial and final projections, so by Lemma $3.3 \phi(x) \tau(x)^{*}$ is in $B \cap B^{*}$. Note that

$$
\left(\phi(x) \tau(x)^{*}\right) \tau(x)=\phi(x) \tau(x)^{*} \tau(x)=\phi(x) \phi\left(x^{*} x\right)=\phi(x) .
$$

Choose a system of matrix units for $A_{v},\left\{e_{i j} \mid 1 \leq i, j \leq m\right\}$, so that $x x^{*}=e_{11}$. Note that $e_{i 1} x \sim x$ and that $\phi\left(e_{i 1} x\right) \tau\left(e_{i 1} x\right)^{*}$ is in $B \cap B^{*}$. Define $V$ by 


$$
V=\sum_{i=1}^{m} \phi\left(e_{i 1} x\right) \tau\left(e_{i 1} x\right)^{*}=\sum_{i=1}^{m} \phi\left(e_{i 1}\right) \phi(x) \tau(x)^{*} \phi\left(e_{1 i}\right) .
$$

As every partial isometry in $B \cap B^{*}$ is order-preserving, $V \in N^{\text {ord }}(B)$. We have $e_{j 1} x \sim x$ and repeating (3) it follows that $V \tau\left(e_{j 1} x\right)=\phi\left(e_{j 1} x\right)$. Similarly, for every $y \in N^{\text {ord }}(A)$ with $y \sim x$, we have $V \tau(y)=\phi(x)$.

Case 2: $v$ has no immediate predecessor and one immediate successor.

Again, this case is dual to Case 1.

Case 3: $v$ has one immediate predecessor and one immediate successor.

Let $u$ be the predecessor and $w$ the successor. First, we show that it suffices to find a partial isometry $V \in B$ with $V^{*} V=V V^{*}=\phi\left(1_{A_{v}}\right)$ so that

$$
V \tau(x)=\phi(x)
$$

for all $x$ minimal elements of $N^{\text {ord }}(A)$ in the equivalence class corresponding to the edge from $v$ to $w$. This condition implies that $\tau(y) V^{*}=\phi(y)$ where $y$ is a minimal element of $N^{\text {ord }}(A)$ in the equivalence class corresponding to the edge from $u$ to $v$. To see this, suppose that $x$ is as above and $y^{*} y=x x^{*}$; then

$$
\phi(y) \phi(x)=\psi(y x)=\tau(y) \tau(x)=\tau(y) V^{*} V \tau(x)=\tau(y) V^{*} \phi(x) .
$$

Since $\phi(x)$ is a partial isometry, it follows that $\tau(y) V^{*}=\phi(y)$.

As before, $V \tau(x)=\phi(x)$ implies that $U=1_{B}-\phi\left(1_{A_{v}}\right)+V$ will satisfy $U \tau(z) U^{*}=\phi(z)$ for all elements $z \in N^{\text {ord }}(A)$ with initial projection in $A_{v}$. Similarly, $\tau(y) V^{*}=\phi(y)$ implies $U \tau(z) U^{*}=\phi(z)$ for all elements $z \in N^{\text {ord }}(A)$ with final projection in $A_{v}$. It remains only to find $V$ satisfying (4). However, we can now repeat the argument of Case 1.

Remark 3.5. It is possible to prove an alternate form of this theorem, where we drop the hypothesis that the digraph of $B$ is chordal. We then conclude that there is an automorphism of $B, \rho$, that $\rho\left(N^{\text {ord }}(B)\right)=N^{\text {ord }}(B)$ and $\tau=\rho \circ \phi$.

We can now prove the main result of this paper, that the fixed-point algebraic order is a complete invariant for a natural family of limit algebras.

Theorem 3.6. Let $\mathcal{A}=\lim \left(A_{k}, \alpha_{k}\right)$ and $\mathcal{B}=\lim _{\rightarrow}\left(B_{k}, \beta_{k}\right)$ be inductive limits of algebras with chordal digraphs and order-preserving maps.

Then there is a *-extendible isomorphism $\Phi: \mathcal{A} \rightarrow \mathcal{B}$ if and only if there is a scale-preserving ordered-group isomorphism $\Theta: K_{0}\left(\mathcal{A} \cap \mathcal{A}^{*}\right) \rightarrow K_{0}\left(\mathcal{B} \cap \mathcal{B}^{*}\right)$ so that $\Theta^{(3)}\left(S_{f}(\mathcal{A})\right)=S_{f}(\mathcal{B})$ and $\Theta$ induces an isomorphism between $K_{0} \mathrm{C}^{*}(\mathcal{A})$ and $K_{0} \mathrm{C}^{*}(\mathcal{B})$.

Proof. This proof is a typical intertwining argument; other examples for limit algebras can be found in $[16,3,5,19]$.

One direction is trivial. For the other, suppose $\Theta$ is an ordered-group isomorphism with the required properties. Then, after possibly restricting to subsystems, we obtain a 
commuting diagram of the form:

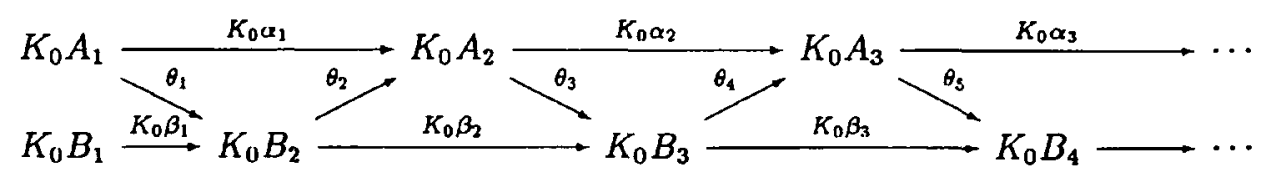

where the $\theta_{i}$ are ordered-group injections preserving $S_{f}\left(A_{i}\right), S_{f}\left(B_{i}\right)$ and having extensions to $K_{0} \mathrm{C}^{*}\left(A_{i}\right)$ and $K_{0} \mathrm{C}^{*}\left(B_{i}\right)$.

By Theorem 3.4, $\theta_{1}$ lifts to an order-preserving $*$-extendible injection $\eta_{1}: A_{1} \rightarrow B_{2}$. Similarly, $\theta_{2}$ lifts to $\zeta_{1}: B_{2} \rightarrow A_{3}$.

As $\zeta_{1} \circ \eta_{1}$ is an order-preserving *-extendible injection with $K_{0}\left(\zeta_{1} \circ \eta_{1}\right)=\theta_{2} \circ \theta_{1}=$ $K_{0} \alpha_{1}$, by the uniqueness part of Theorem 3.4, there is some $U \in A_{2} \cap A_{2}^{*}$ so that

$$
\operatorname{Ad} U \circ \zeta_{1} \circ \eta_{1}=\alpha_{1}
$$

Replacing $\zeta_{1}$ with $\operatorname{Ad} U \circ \zeta_{1}$, we have built the first triangle of the diagram:

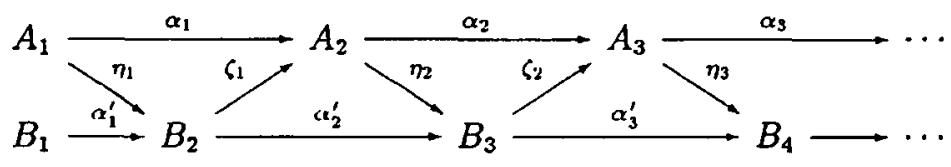

Continuing in this way, we can build this commuting diagram. Applying the universal property of inductive limits then gives the required isomorphism $\Phi$.

\section{Intrinsic characterisation}

We give an intrinsic characterisation of the limit algebras considered in the previous section. In [18], Thelwall showed that a limit algebra is the direct limit of chordal digraph algebras if and only if it has chordal spectrum. To characterise the condition that the embeddings are order-preserving, we need the notion of a covering algebra from [4].

Throughout this section, we assume that $\mathcal{A}$ is a subalgebra of an AF C"-algebra $\mathcal{C}$ that contains a canonical masa $\mathcal{D}$ and that $X$ is the maximal ideal space of $\mathcal{D}$. For $p$ a projection in $\mathcal{D}$, let $\hat{p}$ denote $\{x \in X \mid x(p)=1\}$. Each $c \in N_{\mathcal{D}}(\mathcal{C})$ induces a partial homeomorphism $h_{c}$, from $\widehat{c c^{*}}$ to $\widehat{c^{*} c}$, where $h_{c}(x)$ is $d \mapsto x\left(c d c^{*}\right)$. If $\hat{c}$ denotes the graph of $h_{c}$ in $X \times X$, then we can define

$$
R(\mathcal{A})=\bigcup\left\{\hat{c}: c \in N_{\mathcal{D}}(\mathcal{A})\right\}
$$


Topologise $R(\mathcal{A})$ by using as a basis of open sets $\hat{c}$ for $c \in N_{\mathcal{D}}(\mathcal{A})$. We call $R(\mathcal{A})$ the spectrum of $\mathcal{A}$. Its key property is that it is a complete invariant for the pair $(\mathcal{A}, \mathcal{D})$, up to isometric isomorphism; see [14] or [16, Chapter 7].

Write $\mathcal{A}$ as $\lim _{\rightarrow}\left(A_{j}, \alpha_{j}\right)$ and choose matrix units systems for each $A_{j}$ so that matrix

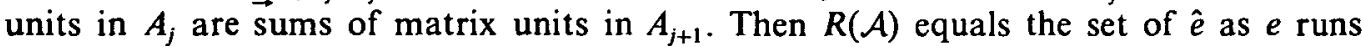
over the matrix units in all the $A_{j}$ and we obtain the same topology if we use as basis only the $\hat{e}$ where $e$ is a matrix unit [16, Chapter 7].

A regular subalgebra of $\mathcal{A}$ is a subalgebra $B$ containing a masa $D$ so that the inclusion $B \rightarrow \mathcal{A}$ sends $N_{D}(B)$ into $N_{D}(\mathcal{A})$; in particular, the matrix units of $B$ are in $N_{\mathcal{D}}(\mathcal{A})$.

Definition 4.1. Suppose $Y$ is a finite subset of $X$. We call a finite dimensional regular subalgebra of $\mathcal{A}, B$, a covering algebra for $\left.R(\mathcal{A})\right|_{Y_{\times Y}}$ if

(1) there is an isomorphism of digraphs $\phi:\left.G(B) \rightarrow R(\mathcal{A})\right|_{Y \times Y}$, and

(2) after identifying $G(B)$ with a system of matrix units, $\phi(e) \in \hat{e}$ for all $e$ in $G(B)$.

The following lemma is proved in [4] but for the reader's convenience we repeat the proof here.

Lemma 4.2. If $Y$ is a finite subset of $X$, then we can find a covering algebra for $\left.R(\mathcal{A})\right|_{Y \times Y}$.

Conversely, if $B$ is a regular digraph subalgebra of $\mathcal{A}$, then there is a finite subset of $X$, call it $Y$, so that there is an injection from $G(B)$ to $\left.R(\mathcal{A})\right|_{Y \times Y}$.

Proof. Let $\mathcal{A}=\lim _{\rightarrow}\left(A_{i}, \alpha_{i}\right)$. As usual, we identify the $A_{i}$ with subalgebras of $\mathcal{A}$ when convenient.

Since the topology in $R(\mathcal{A})$ separates points, we can find a $k$ so that for each diagonal matrix unit $e$ in $A_{k}$, $\hat{e}$ contains at most one point of $Y$. By increasing $k$, we can arrange that each point of $\left.R(\mathcal{A})\right|_{Y \times Y}$ is in the graph of some matrix unit in $A_{k}$. If we let $B \subset A_{k}$ be the span of the matrix units in $A_{k}$ that contain a point in $\left.R(\mathcal{A})\right|_{Y \times Y}$ then it is easy to check that $G(B)$ is isomorphic to $\left.R(\mathcal{A})\right|_{Y_{\times} Y}$ and that the edge associated to a matrix unit in $B$ is sent to a point in the graph of that matrix unit. Being a span of normalising matrix units, $B$ is a regular digraph subalgebra of $\mathcal{A}$.

Conversely, if $B$ is a regular digraph subalgebra of $\mathcal{A}$, then there is a system of normalising matrix units $\left\{e_{i j}\right\}$ for $C^{*}(B)$ so that $B$ is the span of the matrix units that it contains. Let $y \in R(\mathcal{A})$ be an element of $\hat{e}_{11}$ and let

$$
Y=\left\{h_{c}(y): c=e_{i j} \text { for some } j \text { with } e_{j j} \in B\right\} .
$$

Clearly, there is a bijection between $Y$ and the set of minimal diagonal projections of $B$. It follows that there is an injection from $G(B)$ into $\left.R(\mathcal{A})\right|_{Y \times Y}$. 
In [5], the following conditions are shown to be equivalent:

(1) the closed span of $N_{D}^{\text {ord }}(\mathcal{A})$ is $\mathcal{A}$,

(2) $R(\mathcal{A})=\cup\left\{\hat{e}: e \in N_{\mathcal{D}}^{\text {ord }}(\mathcal{A})\right\}$, and

(3) $A=\lim \left(A_{k}, \alpha_{k}\right)$ with $\mathcal{D}$ the limit of the diagonal matrices of the $A_{k}$, and where for any $i, j$ with $i<j, \alpha_{j} \circ \cdots \circ \alpha_{i}$ is locally order-preserving.

Using Lemma 4.2, we can add another condition to this list.

Proposition 4.3. Conditions (1) to (3) above are equivalent to

(4) for each $Y$ a finite subset of $X$, there is a covering subalgebra for $\left.R(\mathcal{A})\right|_{Y \times Y}, B$, with the injection $B \rightarrow \mathcal{A}$ locally order-preserving.

Proof. $(3 \Rightarrow 4)$ This is immediate from the proof of Lemma 4.2, as by (3) we can arrange that the matrix units of each $A_{k}$ are contained in $N_{\mathcal{D}}^{\text {ord }}(\mathcal{A})$.

(4 $\Rightarrow$ ) If $(x, y) \in R(\mathcal{A})$, then set $Y=\{x, y\} \subset X$ and apply (4) to obtain $B$, either $T_{2}$ or $M_{2}$, with the inclusion $B \subset \mathcal{A}$ locally order-preserving. Letting $e \in N_{\mathcal{D}}^{\text {ord }}(\mathcal{A})$ be the $(1,2)$ matrix unit of $B$, it follows by the covering condition that $(x, y) \in \hat{e}$.

The following theorem characterises a somewhat smaller family of limit algebras and canonical masas, those pairs which have a presentation where all the maps are order-preserving. See [5, p. 372] for an example of an algebra and canonical masa not in this family which is nonetheless spanned by $N_{\mathcal{D}}^{\text {ord }}(\mathcal{A})$.

Theorem 4.4. For $\mathcal{A}$ a subalgebra of an $A F \mathrm{C}^{*}$-algebra containing a canonical masa $\mathcal{D}$, the following are equivalent:

(1) there is a presentation of $\mathcal{A}, \underset{\lim }{\rightarrow}\left(A_{k}, \alpha_{k}\right)$, where the $\alpha_{k}$ are order-preserving, and

(2) for each $Y$ a finite subset of $X$, there is a covering algebra for $\left.R(\mathcal{A})\right|_{Y_{\times} Y}$ with the injection $B \rightarrow \mathcal{A}$ order-preserving.

Proof. $(1 \Rightarrow 2)$ The proof is the same as that of $(3 \Rightarrow 4)$ in Proposition 4.3 , save only that the inclusion is order-preserving, and hence its restriction to $B$ is also orderpreserving.

( $2 \Rightarrow 1$ ) Using some presentation of $\mathcal{A}$, it is easy to construct a sequence of nested finite subsets of $X,\left\{Y_{k}\right\}$, so that if $Y=\cup Y_{k}$ then $\left.R(\mathcal{A})\right|_{Y_{\times} Y}$ is dense in $R(\mathcal{A})$. By (2), for each set $Y_{k}$, there is a finite dimensional subalgebra $B_{k}$ of $\mathcal{A}$, with the inclusion $B_{k} \rightarrow \mathcal{A}$ order-preserving. Replacing $B_{k}$ by the algebra generated by $B_{1}, \ldots, B_{k}$, we may assume that the $B_{k}$ are nested. Note that the $B_{k}$ are still finite dimensional subalgebras and the inclusions $B_{k} \rightarrow \mathcal{A}$ are still order-preserving. However, the inclusion $B_{k} \rightarrow B_{k+1}$ may not be order-preserving and to correct this we must enlarge each $B_{k}$.

Let $A_{k}=\operatorname{span}\left\{y, x y x^{*}, x^{*} y x: x, y \in N_{p \cap B_{k}}^{\text {ord }}\left(B_{k}\right)\right\}$. As $B_{k-1} \subseteq B_{k}$, it follows that $A_{k-1} \subseteq A_{k}$. Since we have only added elements of $N_{\mathcal{D}}^{\text {ord }}(\mathcal{A})$ to $B_{k}$, the inclusion $A_{k} \rightarrow \mathcal{A}$ is order- 
preserving. The key new property of the $A_{k}$ is that if $x, y \in N_{D \cap A_{k}}^{\text {ord }}\left(A_{k}\right)$, then $x y x^{*}, x^{*} y x \in$ $N_{p \cap A_{k}}^{\text {ord }}\left(A_{k}\right)$.

We claim that the inclusion $A_{k-1} \rightarrow A_{k}$ is order-preserving. To see this, note that if $x \in N_{\text {ond }_{k-1}^{\text {ord }}}\left(A_{k-1}\right)$ then $x \in N_{D}^{\text {ord }}(\mathcal{A})$. Thus the only way that $x$ could fail to be in $N_{D \cap A_{k}}^{\text {ord }}\left(A_{k}\right)$ is if there is some $y \in N_{D \cap A_{k}}\left(A_{k}\right)$ so that $x y x^{*}$ or $x^{*} y x$ is not in $A_{k}$. But by the new property of $A_{k}$, no such $y$ exists and so the inclusion is order-preserving.

To show that this gives a presentation of $\mathcal{A}$, it remains only to show that $\mathcal{A}=\overline{\cup A_{k}}=\overline{\cup B_{k}}$. However, by the covering property, the spectrum of $\overline{U B_{k}}$ contains a dense subset of $R(\mathcal{A})$ and being closed, it must equal $R(\mathcal{A})$.

Combining Theorem 4.4 and [18] gives the following characterisation.

Corollary 4.5. Let $\mathcal{A}$ be a subalgebra of an $A F \mathrm{C}^{*}$-algebra that contains a canonical masa $\mathcal{D}$ and let $X$ be the maximal ideal space of $\mathcal{D}$. Then the following are equivalent:

(1) there is a presentation of $\mathcal{A}, \lim \left(A_{k}, \alpha_{k}\right)$, where the $A_{k}$ have chordal digraphs and the $\alpha_{k}$ are order-preserving,

(2) for each $Y$ a finite subset of $X,\left.R(\mathcal{A})\right|_{Y \times Y}$ is a chordal digraph and there is a covering subalgebra for $\left.R(\mathcal{A})\right|_{Y_{X Y}}$ with the injection $B \rightarrow \mathcal{A}$ order-preserving.

\section{REFER ENCES}

1. O. Bratteli, Inductive limits of finite dimensional $\mathrm{C}^{*}$-algebras, Trans. Amer. Math. Soc. 171 (1972), 195-234.

2. K. R. DAvidson, When locally contractive representations are completely contractive, $J$. Funct. Anal. 128 (1995), 186-225.

3. K. R. DAvidson and S. C. Power, Isometric automorphisms and homology for non-selfadjoint operator algebras, Quart. J. Math. Oxford Ser. (2) 42 (1991), 271-292.

4. A. P. Donsig, Dilations of limit algebras and interpolating spectrum, Pacific J. Math., to appear.

5. A. P. Donsig and A. Hopenwasser, Order preservation in limit algebras, J. Funct. Anal. 133 (1995), 342-394.

6. A. P. Donsig and S. C. PowER, The failure of approximate inner conjugacy for standard diagonals in regular limit algebras, Canad. Bull. Math. 39 (1996), 420-428.

7. A. P. Donsig and S. C. Power, Homology for operator algebras IV: On the regular classification of limits of 4-cycle algebras, J. Funct. Anal. 150 (1997), 240-287.

8. M. C. Golumbic, Algorithmic graph theory and perfect graphs (Academic Press, Inc., New York, 1980).

9. A. Hopenwasser and J. R. Peters, Full nest algebras, Illinois J. Math. 38 (1994), 501-520.

10. A. Hopenwasser and S. C. Power, Classification of limits of triangular matrix algebras, Proc. Edinburgh Math. Soc. 36 (1992), 107-121.

11. P. S. Muhly and B. Solel, Representations of chordal subalgebras of von Neumann algebras, Hokkaido Math. J. 18 (1989), 263-271. 
12. V. I. Paulsen, S. C. Power and R. R. Smith, Schur products and matrix completions, J. Funct. Anal. 85 (1989), 151-178.

13. J. R. Peters, Y. T. Poon and B. H. WAGner, Triangular AF algebras, J. Operator Theory 23 (1990), 81-114.

14. S. C. POWER, The classification of triangular subalgebras of AF $\mathrm{C}^{*}$-algebras, Bull. London Math. Soc. 22 (1990), 269-272.

15. S. C. Power, Algebraic order on $K_{0}$ and approximately finite operator algebras, $J$. Operator Theory 27 (1992), 87-106.

16. S. C. Power, Limit algebras (Pitman Research Notes in Mathematics, 278, Longman Scientific and Technical, London, 1992).

17. S. C. POWER, Homology for operator algebras II: Stable homology for non-self-adjoint algebras, J. Funct. Anal. 135 (1996), 233-269.

18. M. Thelwall, Dilation theory for subalgebras of AF algebras, J. Operator Theory 25 (1991), 275-282.

19. B. A. Ventura, Strongly maximal triangular AF algebras, Internat. J. Math. 2 (1991), 567-598.

20. N. E. Wegge-Olsen, $K$-theory and $C^{*}$-algebras (Oxford University Press, Oxford, 1993).

\author{
Department of Pure Mathematics \\ UNIVERSITY OF WATERLOO \\ WATERLOO \\ CANADA, N2L 3G1 \\ Current Address: \\ Department of Mathematics \& Statistics \\ UNIVERSITY OF NEBRASKA-LINCOLN \\ LINCOLN, NE \\ U.S.A. 68588-0323 \\ E-mail: adonsig@math.unl.edu
}

